Aluísio de Oliveira ${ }^{\mathrm{a}, \mathrm{c}}$

(D) http://orcid.org/0000-0002-1578-654X

Teresa Cristina Nathan Outeiro Pinto ${ }^{b, c}$ iD https://orcid.org/0000-0003-1470-4976

a Instituto Federal de Educação, Ciência e Tecnologia do Sudeste de Minas Gerais. Juiz de Fora, MG, Brasil.

${ }^{\text {b }}$ Fundação Jorge Duprat Figueiredo de Segurança e Medicina do Trabalho (Fundacentro). Coordenação de Higiene do Trabalho. São Paulo, SP, Brasil.

'Fundação Jorge Duprat Figueiredo de Segurança e Medicina do Trabalho (Fundacentro). Programa de Pós-Graduação Trabalho, Saúde e Ambiente. São Paulo, SP, Brasil.

Contato:

Aluísio de Oliveira

E-mail:

aluisio.oliveira@ifsudestemg.edu.br

Os autores declaram que o estudo não foi subvencionado e que não há conflitos de interesses.

Artigo com base na dissertação de mestrado intitulada Estudo de caso: análise da eficácia de um sistema de ventilação local exaustora utilizado para controle da sílica em uma indústria de borracha do Estado de São Paulo, de Aluísio de Oliveira, defendida em 2016 no Programa de Pós-Graduação stricto sensu Trabalho, Saúde e Ambiente, da Fundação Jorge Duprat Figueiredo de Segurança e Medicina do Trabalho Fundacentro.

Os autores informam que a dissertação que deu base a este trabalho foi apresentada na XI Semana da Pesquisa Atuação da Fundacentro nestes 50 anos: história e perspectivas em SST, realizada em São Paulo, em 2016.

\section{Avaliação da eficácia de sistema de ventilação local exaustora utilizado no controle de sílica cristalina em indústria de borracha de silicone}

\author{
Evaluation of the effectiveness of a local exhaust ventilation \\ system employed for controlling crystalline silica in a silicone \\ rubber industry
}

\section{Resumo}

Introdução: os sistemas de ventilação local exaustora (SVLE) podem ser utilizados para controlar a exposição ocupacional a agentes químicos. Contudo, muitas vezes são indevidamente concebidos, instalados ou operados, tornandose ineficazes. Objetivo: avaliar a eficácia de um SVLE empregado para controle da sílica cristalina utilizada na produção da borracha de silicone. Métodos: a avaliação aplicada foi baseada em procedimentos técnicos descritos em literatura referencial de ventilação industrial e sistematizada em três etapas inspeção inicial, avaliação dos parâmetros do SVLE e avaliação ambiental. Foi realizado um teste de campo em um SVLE instalado em misturador de sílica e silicone utilizado em uma empresa de fabricação de borracha de silicone no Estado de São Paulo. Resultados: as etapas de inspeção e avaliação dos parâmetros do SVLE apontaram deficiências no projeto, na operação e na manutenção do sistema, referendadas por sua reduzida velocidade de captura. Na avaliação ambiental, foram encontradas concentrações de sílica cristalina na fração respirável acima do valor de referência adotado. Conclusão: o estudo ratificou a necessidade de projeto, instalação, operação e manutenção dos SVLE estarem em consonância com os princípios de engenharia de ventilação a fim de se obter o funcionamento eficaz do sistema.

Palavras-chave: ventilação local exaustora; eficácia; borracha; sílica cristalina.

\begin{abstract}
Introduction: local exhaust ventilation (LEV) systems can be used for controlling chemical occupational exposure. However, sometimes they are improperly designed, installed or operated, becaming ineffective. Objective: to evaluate the effectiveness of a LEV used for controlling crystalline silica employed in the production of silicone rubber. Methodology: the assessment carried out was based on technical procedures described in the industrial ventilation reference literature and was systematized in three steps - initial inspection, evaluation of LEV parameters and environmental assessment. A field test was applied in a LEV installed in a silica and silicone mixer of a rubber manufacturing company located in the state of São Paulo, Brazil. Results: the inspection and evaluation steps of the LEV parameters pointed out deficiencies in the design, operation and maintenance of the system, testified by its reduced capture velocity. Regarding the environmental assessment, concentrations of crystalline silica above the reference value were found in the respirable fraction. Conclusion: the study confirmed that, for the LEV effective functioning, their design, installation, operation and maintenance have to be in conformity with the principles of ventilation engineering.
\end{abstract}

Keywords: local exhaust ventilation; effectiveness; rubber; crystalline silica. 


\section{Introdução}

A borracha, em virtude de suas propriedades e grande versatilidade de utilização, pode ser considerada indispensável para a sociedade moderna, sendo utilizada em praticamente todos os setores da economia, principalmente nos segmentos de transportes, indústria química, engenharias e indústrias elétrica e eletrônica ${ }^{1}$.

Dados da Federação das Indústrias do Estado de São Paulo (Fiesp) mostram que a indústria de transformação dos produtos de borracha e plásticos empregou 455.677 trabalhadores formais em $2014^{2}$. Contudo, apesar de sua relevância econômica, o processo produtivo da indústria de transformação da borracha pode expor os trabalhadores a relevantes agentes perigosos à saúde ${ }^{3}$, como material particulado, gases, vapores e outras inúmeras substâncias químicas provenientes dos processos de transformação dos elastômeros ${ }^{4}$. A exposição se dá, predominantemente, pelas vias aéreas (inalação), ou pela via cutânea ${ }^{5}$.

Ao longo dos anos, novos produtos químicos foram incorporados à indústria da borracha devido a necessidade de aprimoramento da qualidade dos produtos, bem como de redução dos custos do processo produtivo $^{6}$. No entanto, foram observadas falhas no âmbito da higiene industrial, com possíveis efeitos sobre a saúde dos trabalhadores. Agravando ainda mais esse cenário, para a fabricação de artefatos de borracha, indústrias no Estado de São Paulo vêm adicionando, ao silicone, quartzo moído contendo mais de $98 \%$ de sílica cristalina. Esse procedimento gera intensa exposição à poeira com alta concentração de sílica cristalina ${ }^{7}$.

Muitas doenças e agravos à saúde dos trabalhadores estão associados à exposição ocupacional à sílica cristalina respirável, dentre os quais se destacam a silicose e a tuberculose pulmonar ${ }^{8}$. Dados da International Agency for Research on Cancer (IARC), integrante da Organização Mundial da Saúde (OMS), classificam a sílica inalada na forma de quartzo ou cristobalita no Grupo 1, ou seja, carcinogênica para seres humanos ${ }^{9}$.

De acordo com o Ministério Público do Trabalho em São Paulo (MPT-SP), há 178 empresas no estado que utilizam a sílica em seus processos produtivos, sobretudo na região do Grande ABC. Ainda segundo o órgão, 44 empresas estavam sob investigação em $2015^{10}$.

A partir da intervenção do MPT-SP, muitas dessas empresas começaram a implantar medidas de controle de riscos em seus ambientes laborais visando à preservação da saúde dos trabalhadores. A mais comum e principal medida de controle adotada nessas empresas foi a implantação de sistemas de ventilação local exaustora (SVLE). O propósito desses sistemas é que o ar poluído seja capturado diretamente (ou próximo) da sua fonte de emanação para evitar que os poluentes alcancem a zona de respiração do trabalhador ${ }^{11}$.

Um sistema de ventilação local exaustora é composto, basicamente, de quatro elementos: captores, rede de dutos, coletor de partículas e ventilador. Quando devidamente concebidos, implantados e operados, são a aplicação mais eficaz para controlar a exposição ocupacional a produtos químicos nocivos, assegurando a proteção do trabalhador ${ }^{12}$. Um SVLE eficaz pode neutralizar ou, em alguns casos, até mesmo eliminar o risco ${ }^{13}$.

Entretanto, é possível que em parte dessas empresas os sistemas de ventilação não tenham sido devidamente concebidos, implantados ou operados e, consequentemente, não sejam capazes de reduzir a exposição ocupacional à sílica cristalina a níveis aceitáveis para padrões de saúde. Para a Associação Brasileira de Normas Técnicas (ABNT), eficácia é a extensão na qual atividades planejadas são realizadas e resultados planejados são alcançados ${ }^{14}$. Por conseguinte, a eficácia de um SVLE pode ser associada à sua capacidade de remover poluentes, gerados pelo processo de produção, do ambiente onde está instalado. A redução dos poluentes deve, obrigatoriamente, atingir os valores previstos no projeto do sistema, para que suas concentrações no ambiente de trabalho fiquem abaixo dos limites máximos previstos na legislação, caso contrário o sistema não será considerado eficaz.

Esta pesquisa teve como objetivo avaliar a eficácia de um Sistema de Ventilação Local Exaustora (SVLE) empregado para controle da sílica cristalina utilizada na produção da borracha de silicone.

\section{Métodos}

Estudo de caso realizado no ambiente fabril de uma empresa de artefatos de borracha localizada no Estado de São Paulo. A empresa, composta por matriz e filial, possuía produção diversificada, direcionando sua capacidade produtiva conforme as necessidades do mercado. Os principais segmentos econômicos atendidos pela empresa eram: automobilístico, linha branca e utilidades domésticas.

O processo produtivo da empresa era dividido entre as unidades filial e matriz, sendo a primeira responsável pela preparação dos insumos (borracha e borracha de silicone) a serem utilizados na matriz para fabricação do produto final. Em virtude das especificidades da dinâmica do processo e das substâncias utilizadas para preparação dos insumos, este estudo foi realizado nas dependências da filial, após obter autorização da direção da empresa para realizar a pesquisa em suas instalações.

Para conhecer seu processo produtivo e coletar dados qualitativos e quantitativos, foram realizadas 
quatro visitas à empresa, de aproximadamente seis horas cada, em dias alternados, durante o mês de agosto de 2015.

A coleta de dados foi feita por registro de anotações resultantes de observação direta não participativa e por meio de registros fotográficos e de vídeos. Os dados levantados e coletados foram utilizados exclusivamente para esta pesquisa, sendo mantido o sigilo da empresa participante e a proteção à privacidade dos trabalhadores, conforme preceitos da Resolução no 466/2012, do Conselho Nacional de Saúde ${ }^{15}$.

\section{Análise da eficácia do sistema de ventilação local exaustora}

O método de avaliação da eficácia do SVLE aplicado neste trabalho foi sistematizado em consonância com os preceitos estabelecidos nos seguintes manuais de ventilação industrial: o Industrial ventilation: a manual of recommended practice da American
Conference of Governmental Industrial Hygienists (ACGIH $)^{12}$, dos Estados Unidos, e o Controlling airborne contaminants at work: A guide to local exhaust ventilation (LEV) da Health and Safety Executive $(\mathrm{HSE})^{16}$, do Reino Unido, referências no assunto.

Neste estudo, foi avaliada a eficácia do SVLE instalado no misturador de massa da empresa. A avaliação envolveu processos qualitativos e quantitativos, sistematizados em três etapas detalhadas a seguir:

\section{Etapa 1 - Inspeção inicial}

Nesta fase, por meio de observações diretas in loco e de contatos com representantes da empresa para esclarecimento de dúvidas, foram levantadas informações sobre o processo produtivo da empresa a fim de compreender o contexto que levou à implantação do sistema de ventilação. A inspeção inicial envolveu ainda a análise técnica dos componentes do sistema e da sua dinâmica de operação, conforme orientações descritas no Quadro 1.

Quadro 1 Itens de inspeção do Sistema de Ventilação Local Exaustora

\begin{tabular}{|c|c|}
\hline \multirow{8}{*}{ SVLE } & Possui projeto de concepção e instalação? \\
\hline & Possui informações técnicas do sistema ou dos componentes? \\
\hline & A instalação do sistema foi realizada por empregados da empresa ou por empresa especializada? \\
\hline & Possui programa de manutenção? \\
\hline & A manutenção do sistema é realizada por empregados da empresa ou terceirizada? \\
\hline & A manutenção é realizada por profissional qualificado? \\
\hline & Há histórico das intervenções realizadas no sistema? \\
\hline & O sistema possui funcionamento contínuo? \\
\hline \multirow{5}{*}{ Captores } & Estão instalados em todos os pontos de geração/liberação de poluentes? \\
\hline & Os tipos instalados são adequados? \\
\hline & As instalações (distância da fonte, posição e conexões) estão corretas? \\
\hline & Existência de pontos de obstrução ou de fluxo cruzado de ar em relação à aspiração do captor? \\
\hline & Presença de avarias (superfícies desgastadas, corroídas ou amassadas)? \\
\hline \multirow{4}{*}{ Dutos } & O traçado da rede é adequado? \\
\hline & Presença de pontos de vazamento ou obstrução? \\
\hline & Má fixação das singularidades da rede? \\
\hline & Há pontos para medição de velocidade ou pressão? \\
\hline \multirow{6}{*}{ Filtros } & Estão instalados corretamente? \\
\hline & Os tipos empregados estão corretos? \\
\hline & Presença de avarias? \\
\hline & Existem procedimentos padronizados para substituição ou limpeza? \\
\hline & Utilização de EPI durante esses procedimentos? \\
\hline & Destinação dos resíduos do sistema? \\
\hline \multirow{5}{*}{ Ventiladores } & O tipo empregado está correto? \\
\hline & Indícios de desgaste ou corrosão? \\
\hline & Sentido de rotação está correto? \\
\hline & Deslizamento no sistema de acionamento? \\
\hline & Presença de ruído ou vibração anormal? \\
\hline
\end{tabular}

Fonte: adaptado de Department of Occupational Safety and Health. Guidelines on occupational safety and health for design, inspection, testing and examination of local exhaust ventilation system. Malaysia: Ministry of Human Resources, $2008^{17}$. 


\section{Etapa 2 - Avaliação dos parâmetros do SVLE}

Nesta etapa foram realizados os testes de medição da velocidade de captura do sistema e os ensaios de visualização do fluxo de ar capturado.

A velocidade de captura de um sistema de ventilação é definida como a velocidade mínima necessária para indução dos poluentes do seu ponto de geração ou liberação para o interior do captor ${ }^{12}$.

A medição da velocidade de captura é uma poderosa ferramenta na avaliação de sistemas ventilação, uma vez que expressa uma medida direta e quantitativa do desempenho do sistema. Por sua vez, os marcadores visuais (tubo de fumaça), por meio da geração de fumaça na zona de captação do sistema, permitem a visualização da movimentação geral do fluxo de ar nessa região. Por isso, esses parâmetros são comumente utilizados no processo de avaliação da eficácia de sistemas de ventilação ${ }^{16,18}$.

Para avaliação da velocidade de captura do SVLE implantado no misturador de sílica e silicone, foi utilizado um termoanemômetro compacto, marca TESTO, modelo 435, com sonda integrada de velocidade e temperatura, punho telescópico e resolução de $0,01 \mathrm{~m} / \mathrm{s}$. Para obtenção da velocidade de captura, a área da seção transversal da câmara do misturador foi segmentada em retângulos de áreas iguais. No centro de cada retângulo foram efetuadas medições pontuais de velocidade. A média aritmética do conjunto dos valores pontuais de velocidade encontrados em cada medição corresponde à velocidade de captura do sistema ${ }^{13,16}$.

Para visualização do fluxo de ar capturado pelo sistema de ventilação na face da câmara do misturador e, também, para localização de possíveis pontos de perda de carga (vazamentos), foi utilizado o flow check da marca Dräger. Este instrumento contém em seu interior um sistema de geração de calor responsável pelo aquecimento da mistura especial de álcoois (fluido não tóxico) presente na ampola inserida no interior do equipamento. Pela variação de densidade, o fluido escapa do instrumento, condensando-se ao entrar em contato com o ar ambiente ${ }^{19}$, gerando fumaça, que escapará por eventuais pontos de vazamento, o que permite que eles sejam identificados visualmente.

Todos os instrumentos utilizados nos testes do sistema de ventilação foram devidamente calibrados e inspecionados.

\section{Etapa 3 - Avaliação ambiental}

Na última etapa, foi avaliada a concentração de sílica cristalina na fração respirável na região do posto de trabalho do misturador de sílica e silicone, e nas proximidades dos filtros do SVLE. De acordo com Ojima ${ }^{20}$, a eficácia de um SVLE pode ser determinada a partir da avaliação da concentração de poeira de sílica cristalina presente na área de captação do sistema.

A avaliação ambiental foi realizada em consonância com os preceitos da Norma de Higiene Ocupacional no 08 (NHO-08): "Coleta de Material Particulado Sólido Suspenso no Ar de Ambientes de Trabalho"21. A coleta das amostras foi realizada por um sistema próprio, composto por bomba de amostragem, mangueira, dispositivos de coleta e filtros.

Para coleta das amostras foram utilizadas bombas da marca Buck Genie, modelo VSS-5, calibradas com vazão de $2,75 \mathrm{~L} / \mathrm{min}$, conforme determinações do manual do fabricante do ciclone GS-3 Multiple-inlet Cyclone. A calibração das bombas foi realizada com o calibrador eletrônico Gilian Gilibrator 2, da marca Sensidyne $^{22}$. O processo de calibração foi realizado antes e depois de cada amostragem concluída, sendo permitida a variação máxima de $\pm 5 \%$ do valor da calibração inicial. As amostras que apresentaram variação maior foram descartadas, conforme recomendações da NHO-07: "Calibração de Bombas de Amostragem Individual pelo Método da Bolha de Sabão"23.

Para coleta da fração de sílica cristalina respirável foi utilizado filtro de membrana de PVC com $37 \mathrm{~mm}$ de diâmetro e $5 \mu \mathrm{m}$ de poro. $\mathrm{O}$ dispositivo de coleta foi montado com suporte, porta filtro e separador de partículas do tipo ciclone da marca SKC, modelo GS-3 Multiple-inlet Cyclone ${ }^{22}$.

Segundo a NHO-08 21 , a avaliação da eficácia de medidas coletivas de controle (no caso do SVLE instalado no misturador) deve ser realizada por meio de coleta estática, ou seja, com o equipamento coletor posicionado em ponto fixo. Neste caso, portanto, não se utiliza a amostragem individual, que representa a exposição individual do trabalhador ${ }^{21,24}$. As concentrações obtidas por meio dessa estratégia de amostragem são, então, comparadas com o limite de exposição ocupacional média ponderada no tempo (TLV-TWA) da ACGIH ${ }^{25}$, para sílica cristalina respirável ( $\alpha$ quartzo e cristobalita), igual a $0,025 \mathrm{mg} / \mathrm{m}^{3}$.

As amostras foram coletadas cobrindo, no mínimo, 70\% da jornada de trabalho de 8 horas diárias, conforme procedimentos descritos na NHO-08 ${ }^{21}$, durante quatro dias não consecutivos.

Após coletadas as amostras, a determinação da concentração de sílica cristalina respirável foi realizada por meio dos procedimentos descritos na NHO-03: "Análise Gravimétrica de Aerodispersóides Sólidos Coletados sobre Filtros de Membrana” e pelo 
método: "Determinação quantitativa de sílica cristalina por difração de raios X" 26,27 .

\section{Resultados e Discussão}

O SVLE foco da pesquisa foi instalado na empresa após inspeção do MPT-SP. A implantação do sistema, bem como a adoção de outras medidas de controle, fez parte de um conjunto de ações adotadas pela empresa para preservação da saúde e segurança dos trabalhadores, conforme obrigações previstas no Termo de Ajuste de Conduta (TAC) firmado com o MPT-SP.

Serão apresentados os resultados das três etapas de avaliação: Etapa 1 - inspeção inicial, ou seja, informações técnicas do SVLE, tanto de caráter geral (especificação, instalação, operação e manutenção do sistema de ventilação), como de caráter específico (análise dos elementos do SVLE: captores, dutos, ventilador, motor de acionamento e filtros); Etapa 2 - avaliação dos parâmetros do SVLE; Etapa 3 - avaliação ambiental, realizada com a finalidade de verificar se a implantação do SVLE mantém as concentrações de poeira contendo sílica cristalina dentro dos parâmetros recomendados.

Etapa 1 - Especificação, instalação, operação e manutenção do sistema de ventilação

O SVLE foi especificado pela empresa terceirizada responsável pela prestação dos serviços de segurança e medicina do trabalho da fábrica. Entretanto, sua instalação foi realizada pelos próprios empregados da fábrica de borracha, conforme orientações da empresa terceirizada.

Durante as observações iniciais, constatou-se que a empresa não possuía plano de operação e manutenção do sistema de ventilação instalado, havia apenas a orientação para troca dos filtros a intervalos regulares de três meses. Sem plano de manutenção operacional e preventiva, somente manutenções corretivas eram realizadas, pelos próprios empregados da fábrica, em momentos de parada ou de funcionamento irregular do SVLE.

Nesse cenário, revelaram-se as inconformidades iniciais do sistema instalado, uma vez que um SVLE deve ser dimensionado, implantado e operado por profissionais qualificados, sempre em conformidade com as normas e boas práticas de engenharia de ventilação ${ }^{16,28}$. Destaca-se que, após o dimensionamento e a implantação, testes regulares são indispensáveis para garantia da eficácia do SVLE, caso contrário, o sistema pode apresentar falhas durante sua operação $0^{29}$.
Os resultados obtidos mostraram que o sistema de ventilação implantado na empresa possuía deficiências em seu projeto de concepção e instalação, o que impactava negativamente seu desempenho, como veremos a seguir:

\section{Captores}

Constatou-se que o sistema de captura estava acoplado de forma inadequada à estrutura do misturador e em somente um de seus lados. Verificou-se, também, que o ponto de captura do sistema estava distante da região de abastecimento da máquina, ou seja, da zona de maior dispersão da poeira de quartzo, e que o sistema possuía um ponto com má vedação, acarretando lançamento da sílica no ambiente da fábrica. Além dos problemas citados anteriormente, verificou-se que a ascensão da prensa da máquina causava obstrução parcial do ponto de captura do sistema de ventilação, prejudicando o processo de captação da poeira de quartzo dispersa na zona de alimentação da máquina e o aumento da exposição dos trabalhadores.

\section{Dutos}

A rede de dutos do SVLE, em toda sua extensão, era formada por duto flexível (tipo "traqueia") e continha alguns pontos de estrangulamento por deformação, podendo interferir no funcionamento adequado do equipamento. Conforme menciona a ACGIH $^{12}$, dutos flexíveis podem aumentar as perdas de carga do sistema, uma vez que são mais sucestíveis à deformação acidental. Constatou-se, também, que a rede de dutos não possuía pontos de acesso para inspeção, medição de velocidade e limpeza, conforme recomendado pela HSE ${ }^{16}$.

\section{Ventilador e motor de acionamento}

A empresa não possuía registros de informações técnicas sobre o motor de acionamento e o ventilador do sistema de ventilação. A falta dessas especificações inviabilizou a avaliação do equipamento empregado, uma vez que o projeto de um ventilador define suas características operacionais em função da especificidade de cada aplicação ou conjunto de aplicações para as quais é dimensionado ${ }^{16}$. Os ventiladores de um SVLE devem ser devidamente dimensionados e instalados, caso contrário poderá ocorrer consumo desnecessário de energia elétrica, operação ruidosa e/ ou ineficácia do sistema de ventilação, prejudicando seu funcionamento e eficácia ${ }^{16,30}$.

\section{Filtros}

O recomendado pela literatura para os filtros de coleta da poeira captada pelo sistema é que eles sejam instalados fora da zona de produção e, também, que 
sejam enclausurados com exaustão $0^{12}$. Os filtros do SVLE analisado foram instalados inadequadamente dentro do ambiente de produção da empresa e sem enclausuramento. Além do local incorreto de instalação, os filtros possuíam má fixação junto aos tambores coletores, o que acarretava vazamento contínuo da poeira coletada para o ambiente, com consequente exposição dos trabalhadores e deposição da poeira no piso e nos equipamentos. A movimentação dos trabalhadores, as práticas de varrição e as correntes naturais ou forçadas de ar provocavam a dispersão dessa poeira depositada, que voltava a se espalhar no ambiente, propiciando aumento da concentração de poeira dispersa no ar.

Os filtros saturados e a poeira coletada eram embalados em sacos e enviados para descarte em aterros sanitários.

Assim, a inspeção inicial apontou uma série de deficiências na especificação, instalação, operação e manutenção do sistema de ventilação, que podiam implicar o funcionamento irregular do sistema. Para verificar essa possibilidade, realizaram-se a avaliação dos parâmetros do SVLE e a avaliação ambiental, cujos resultados apresentamos e comentamos na etapa 2.

\section{Etapa 2 - Avaliação dos parâmetros do SVLE}

A velocidade de captura do sistema, calculada conforme descrito no item Métodos, foi de, apenas, $0,15 \mathrm{~m} / \mathrm{s}$. Observa-se que o sistema de ventilação opera com velocidade muito abaixo de $1 \mathrm{~m} / \mathrm{s}$, valor recomendado pela $\mathrm{HSE}^{16}$. Esse fato demonstra a incapacidade do sistema de ventilação instalado para capturar eficientemente os poluentes, principalmente a sílica, antes que esses atinjam a zona respiratória do trabalhador.

Por meio da utilização do gerador de fumaça flow check, verificou-se que, em virtude da baixa velocidade de captura do sistema, o fluxo de fumaça produzido pelo equipamento sofreu grande dispersão, não sendo adequadamente capturado pelo SVLE.

\section{Etapa 3 - Avaliação ambiental}

Os resultados das avaliações ambientais realizadas para mensurar a concentração de sílica cristalina respirável na região de abastecimento do misturador e nas proximidades dos locais de instalação dos filtros do sistema de ventilação (Tabela 1) apontaram não somente a ineficácia do SVLE instalado, mas também o grande risco de exposição dos que trabalhavam e transitavam nessas regiões.

Os dados apresentados na Tabela 1 expressam a concentração de poeira de quartzo na fração respirável em cada uma das amostras coletadas, obtidas conforme procedimentos estabelecidos na $\mathrm{NHO}-08^{21}$. Por meio da análise desses dados, percebe-se que em todas as coletas, com exceção daquela realizada na região do misturador no segundo dia da avaliação ambiental, os valores de concentração de sílica cristalina respirável encontrados excederam o valor de referência da $\mathrm{ACGIH}^{25}$, adotado nesta pesquisa, ou seja, $0,025 \mathrm{mg} / \mathrm{m}^{3}$.

Assim, os resultados das etapas de avaliação propostas corroboraram entre si, evidenciando deficiências no funcionamento do SVLE.

Tabela 1 Resultados das avaliações ambientais de fração de poeira respirável realizadas na região de abastecimento do misturador de sílica e silicone, e dos filtros do Sistema de Ventilação Local Exaustora (SVLE) instalado para controle de sílica cristalina em fábrica de borracha de silicone, Estado de São Paulo, 2015

\begin{tabular}{|c|c|c|c|c|c|c|}
\hline Dia da amostragem & $\begin{array}{c}\text { Local / região de } \\
\text { amostragem }\end{array}$ & $\begin{array}{c}\text { Código da } \\
\text { amostra }\end{array}$ & $\begin{array}{c}\text { Volume } \\
\text { amostrado } \\
\left(\mathrm{m}^{3}\right)\end{array}$ & $\begin{array}{l}\text { Massa } \\
\text { de poeira } \\
\text { respirável } \\
\text { (mg) }\end{array}$ & $\begin{array}{l}\text { Sílica } \\
\text { cristalina } \\
\text { respirável } \\
\text { (mg) }\end{array}$ & $\begin{array}{c}\text { Concentração de sílica } \\
\text { cristalina respirável } \\
\left(\mathrm{mg} / \mathrm{m}^{3}\right)\end{array}$ \\
\hline \multirow{2}{*}{$1^{\circ}$} & Misturador & $C L-87$ & 0,987 & 0,151 & 0,074 & 0,075 \\
\hline & Filtros & CL-88 & 0,965 & 0,480 & 0,192 & 0,198 \\
\hline \multirow{2}{*}{$2^{\circ}$} & Misturador & CL-98 & 0,983 & 0,081 & $\mathrm{Ml}^{*}$ & - \\
\hline & Filtros & CL-97 & 0,964 & 0,330 & 0,028 & 0,029 \\
\hline \multirow{2}{*}{$3^{\circ}$} & Misturador & $\mathrm{Cm}-17$ & 0,997 & 0,212 & 0,044 & 0,044 \\
\hline & Filtros & $\mathrm{Cm}-30$ & 0,976 & 0,353 & 0,063 & 0,064 \\
\hline \multirow{2}{*}{$4^{\circ}$} & Misturador & $\mathrm{Cm}-35$ & 0,984 & 0,196 & 0,042 & 0,042 \\
\hline & Filtros & $\mathrm{Cm}-43$ & 0,959 & 0,444 & 0,145 & 0,151 \\
\hline
\end{tabular}

*MI = Massa insuficiente (neste dia, o misturador teve seu período de funcionamento reduzido em virtude da falta de silicone. Após a chegada dessa matéria-prima, o tempo de operação foi de apenas 2 horas e 15 minutos, no período da tarde). 


\section{Conclusão}

Com base nos resultados obtidos, constatou-se que a origem das inconformidades do sistema de ventilação avaliado encontrava-se, sobretudo, na sua concepção. O sistema foi agregado ao processo produtivo da empresa, sem que tivesse sido adequadamente projetado em função das particularidades desse processo. Em virtude disso, problemas técnicos foram encontrados, indicando o funcionamento ineficaz do sistema, ocasionado tanto pela velocidade de captura, muito aquém do exigido pela literatura especializada, como pela inadequação do fluxo de ar na zona de captação do sistema de ventilação, possibilitando dispersão do agente contaminante no ambiente.

Consequentemente, as concentrações de sílica cristalina encontradas superaram o valor de referência adotado, o que indica a necessidade de readequação do projeto, da instalação, da operação e da manutenção do sistema de ventilação em consonância com os princípios de engenharia de ventilação, a fim de proporcionar um funcionamento eficaz do sistema e, por conseguinte, a efetiva proteção dos trabalhadores.

O método empregado mostrou-se satisfatório ao tornar possível avaliar o desempenho do SVLE e caracterizá-lo como ineficaz no controle da sílica cristalina respirável dispersa no ambiente de trabalho durante a produção da borracha de silicone. O método empregado fornece subsídios para auxiliar a implantação e manutenção de medidas de controle de agentes químicos em ambientes de trabalho, e possivelmente poderá ser utilizado para avaliar a eficácia de sistemas de ventilação instalados em outras empresas.

\section{Contribuições de autoria}

Oliveira A foi responsável pela construção do projeto, realização da pesquisa, consolidação e análise dos resultados; Pinto TCNO por orientar todo o processo de realização da pesquisa original. Ambos contribuíram igualmente na redação do manuscrito e aprovação da versão final publicada.

\section{Referências}

1. Menda M. Borrachas: química e tecnologia [Internet]. São Paulo: Conselho Regional de Química da 4 Região; 2012 [citado em 2015 jul 8]. Disponível em: http://www.crq4.org.br/ quimicaviva_borrachas

2. Federação das Indústrias do Estado de São Paulo. Panorama da indústria de transformação brasileira [Internet]. São Paulo: Fiesp; 2015 [citado em 2015 nov 15]. Disponível em: http://www.fiesp.com.br/ indices-pesquisas-e-publicacoes/panorama-daindustria-de-transformacao-brasileira/

3. International Labour Office. Chapter 80: Rubber industry. Geneva: Encyclopedia of Occupational Health and Safety; 1998 [citado em 2015 jun 10]. Disponível em: http://www.ilocis.org/documents/ chpt80e.htm

4. Neves H, Moncau, J, Kaufmann P, Wunsch Filho V. Mortalidade por câncer em trabalhadores da indústria da borracha de São Paulo. Rev Saúde Pública. 2006;40(2):271-279.

5. International Agency for Research on Cancer. Occupational exposures in the rubbermanufacturing industry. In: Chemical agents and related occupations: a review of human carcinogens [Internet]. Lyon: Internacional Agency for Research on Cancer; 2012 [citado em 2015 ago 12]. Disponível em: https://monographs.iarc.fr/wpcontent/uploads/2018/06/mono100F.pdf
6. Burgess WA. Identificação de possíveis riscos à saúde do trabalhador nos diversos processos industriais. Belo Horizonte: Ergo Editora; 1997.

7. Fundação Jorge Duprat Figueiredo de Segurança e Medicina do Trabalho. Casos de silicose ocorrem em fábrica de borrachas de silicone [Internet]. 2015 [citado em 2015 set 5]. Disponível em: http://www.fundacentro.gov.br/noticias/detalheda-noticia/2015/7/casos-de-silicose-ocorrem-emfabrica-de-borrachas-de-silicone

8. National Institute for Occupational Safety and Health. Health effects of occupational exposure to respirable crystalline silica [Internet]. Niosh: Cincinnati; 2002 [citado em 2015 set 8]. Disponível em: http://www. cdc.gov/niosh/docs/2002-129/pdfs/2002-129.pdf

9. International Agency for Research on Cancer. Agents classified by the IARC monographs [Internet]. Lyon; 2013 [citado em 2015 ago 11]. Disponível em: https://monographs.iarc.fr/agentsclassified-by-the-iarc/

10. Ministério Público do Trabalho em São Paulo. MPT alerta: Pó de sílica pode matar trabalhadores em questão de meses. 2015 [citado em 2015 set 29]. Disponível em: http://www.prt2.mpt.gov.br/241mpt-alerta-po-de-silica-pode-matar-trabalhadoresem-questao-de-meses

11. Morteza MM, Hossein K, Amirhossein M, Naser $\mathrm{H}$, Gholamhossein H, Hossein F. Controlling 
pollutants in a foundry with exhaust vents. Arh Hig Rada Toksikol. 2013;64:123-131.

12. American Conference of Governmental Industrial Hygienists. Industrial ventilation: A manual of recommended practice for design. $28 \mathrm{ed}$. Cincinnati: ACGIH; 2013.

13. Burgess WA, Ellenbecker MJ, Treitman RD. Ventilation for control of the work environment. New Jersey: Wiley-Interscience; 2004.

14. Associação Brasileira de Normas Técnicas. ABNT NBR ISO 9000:2015: Sistemas de gestão da qualidade: fundamentos e vocabulário [Internet]. [s.d.] [citado em 2015 dez 20]. Disponível em: https://www.abntcatalogo.com.br/norma. aspx?ID= 345040

15. Ministério da Saúde (Brasil). Conselho Nacional de Saúde. Resolução 466/2012 [Internet]. [citado em 2019 jan 15]. Disponível em: http:// bvsms.saude.gov.br/bvs/saudelegis/cns/2013/ res0466_12_12_2012.html

16. Health and Safety Executive. Controlling airborne contaminants at work: a guide to local exhaust ventilation (LEV). Sudbury: HSE Books; 2011.

17. Department of Occupational Safety and Health. Guidelines on occupational safety and health for design, inspection, testing and examination of local exhaust ventilation system [Internet]. Malaysia: Ministry of Human Resources; 2008 [citado em 2014 set 12]. Disponível em: http:// www.dosh.gov.my/images/dmdocuments/glx/ ve_gl_lev.pdf

18. Marlow AD, Looney T, Reutman S. An evaluation of local exhaust ventilation systems for controlling hazardous exposures in nail salons. 2012. EPHB Report;005-164:1-28.

19. Dräger Flow Check [citado em 2014 set 10]. Disponível em: https://www.draeger.com/Products/ Content/flow-check-pi-9046525-en-gb.pdf

20. Ojima J. Efficiency of a Tool-mounted Local Exhaust Ventilation System for controlling dust exposure during metal grinding operations. Ind Health. 2007;45:817-819.

21. Fundação Jorge Duprat Figueiredo de Segurança e Medicina do Trabalho. Coleta de material particulado sólido suspenso no ar de ambientes de trabalho. Procedimento técnico NHO-08. São Paulo; 2007.

22. SKC. Operating Instructions. GS-3 Multipleinlet Cyclone Cat. Nos. 225-100 and 225-103 [Internet]. [s.d.] [citado em 2015 ago 10]. Disponível em: http://www.skcinc.com/catalog/pdf/ instructions/37097.pdf

23. Fundação Jorge Duprat Figueiredo de Segurança e Medicina do Trabalho. Calibração de bombas de amostragem individual pelo método da bolha de sabão. Procedimento técnico NHO-07. São Paulo; 2002.

24. Bon AMT. Exposição ocupacional à sílica e silicose entre trabalhadores de marmorarias, no município de São Paulo. Tese de Doutorado em Saúde Ambiental: Universidade de São Paulo; 2006.

25. American Conference of Governmental Industrial Hygienists. TLVs e BEIs: Baseados na Documentação dos Limites de Exposição Ocupacional (TLVs) para Substâncias Químicas e Agentes Físicos \& Índices Biológicos de Exposição (BEIs). São Paulo: ABHO; 2014.

26. Fundação Jorge Duprat Figueiredo de Segurança e Medicina do Trabalho. Análise gravimétrica de aerodispersóides sólidos coletados sobre filtro de membrana: Método de ensaio. Procedimento técnico NHO-03. São Paulo; 2001.

27. Anjos MA. Determinação quantitativa de sílica cristalina por difração de Raios X. Rev. Bras. Saúde Ocup. 1989;17(65):55-59.

28. Workplace Health and Safety Queensland. Silica: Technical guide to managing exposuwre in the workplace. Work-related disease strategy 20122022 [Internet]. 2013 Feb [citado em 2014 Mar 15]. Disponível em: https://www.worksafe.qld.gov.au/ data/assets/pdf_file/0008/83186/silica_managing_ workplace.pdf

29. Silva JA. Estudo da relação entre as concentrações ambientais das névoas de ácido crômico em processo de eletrodeposição de cromo duro e a eficácia de três diferentes sistemas de proteção coletiva. Dissertação de Mestrado em Saúde Ambiental: Universidade de São Paulo; 2003.

30. Fox RW, McDonald AT, Pritchard PJ. Introdução à mecânica dos fluidos. Rio de Janeiro: LTC; 2014. 